\title{
Endobronchial ultrasound increases the diagnostic yields of polymerase chain reaction and smear for pulmonary tuberculosis
}

\author{
Shu-Min Lin, MD, Yung-Lun Ni, MD, Chih-Hsia Kuo, MD, Ting-Yu Lin, MD, Tsai-Yu Wang, MD, \\ Fu-Tsai Chung, MD, and Han-Pin Kuo, MD, PhD
}

\begin{abstract}
Objectives: Our objective was to determine the contribution of endobronchial ultrasound in the diagnostic yields of acid-fast bacillus smear, nucleic acid amplification tests, and culture in bronchoalveolar lavage fluid for pulmonary tuberculosis.
\end{abstract}

\begin{abstract}
Methods: During a 1-year interval, 99 patients who had initial sputum-negative acid-fast bacillus smears or no sputum but were later proven to have a positive culture for Mycobacterium tuberculosis in their sputum or bronchoalveolar lavage fluid were retrospectively studied. Among them, 56 patients underwent bronchoscopy with endobronchial ultrasound (EBUS group) and 43 patients received conventional bronchoscopy for bronchoalveolar lavage (non-EBUS group).
\end{abstract}

Results: The diagnostic yields of the nucleic acid amplification tests $(89.3 \%, 50 / 56 ; P=.006)$, acid-fast bacillus smear $(30.4 \%, 17 / 56 ; P=.013)$, and $M$ tuberculosis culture in bronchoalveolar lavage fluid $(67.9 \%$, $38 / 56 ; P=.041$ ) were significantly higher in the EBUS group of patients. The results of those who underwent conventional bronchoscopy were $65.1 \%$ (28/43), 9.3\% (4/43), and 46.5\% (20/43), respectively. Combining bronchoalveolar lavage fluid smear and nucleic acid amplification tests, we made a rapid diagnosis of pulmonary tuberculosis in $51(91.1 \%)$ of the 56 EBUS patients and $29(67.4 \% ; P=.004)$ of the 43 non-EBUS patients.

Conclusions: The introduction of endobronchial ultrasound increases the diagnostic yield of the nucleic acid amplification tests, acid-fast bacillus smear, and $M$ tuberculosis culture from bronchioalveolar lavage fluid in patients with pulmonary tuberculosis who have negative sputum smear or no sputum production. (J Thorac Cardiovasc Surg 2010;139:1554-60)

The definitive diagnosis of active pulmonary tuberculosis (TB) usually requires the isolation of Mycobacterium tuberculosis from respiratory secretions. It may take up to 6 weeks to identify $M$ tuberculosis from cultured specimens, ${ }^{1}$ although the detection time may be shortened when automated and semiautomated liquid culture systems are used. ${ }^{2,3}$ Thus, it is common practice to commence early expectant treatment for pulmonary $\mathrm{TB}$ on the basis of clinical evidence even before culture confirmation. Among this clinical evidence, sputum examination is the most useful method for diagnosing active pulmonary TB. ${ }^{4}$ However, between $25 \%$ and $60 \%$ of patients with pulmonary TB have negative sputum smears or no spontaneous sputum production. ${ }^{5}$ Thus, the diagnosis of TB may be missed initially in

\footnotetext{
From the Department of Thoracic Medicine, Chang Gung Memorial Hospital, Chang Gung University, School of Medicine, Taipei, Taiwan.

This project is supported by NSC 97-2314-B-182A-088-MY3, National Science Council, Taiwan, Republic of China.

Disclosures: None

Received for publication Aug 19, 2009; revisions received Nov 22, 2009; accepted for publication Feb 9, 2010.

Address for reprints: Han-Pin Kuo, MD, PhD, Department of Thoracic Medicine, Chang Gung Memorial Hospital, 199 Tun-Hwa N Rd, Taipei, Taiwan (E-mail: q8828@ms11.hinet.net).

0022-5223/\$36.00

Copyright $\Subset 2010$ Published by Elsevier Inc. on behalf of The American Association for Thoracic Surgery

doi:10.1016/j.jtcvs.2010.02.019
}

these patients. This error may incur delays in the treatment of pulmonary TB until the return of positive culture results. As a result, delays in diagnosis and treatment have been recognized as an important cause of death due to pulmonary TB. ${ }^{6}$ The utility of rapid diagnostic tests in the early detection of smear-negative TB is, therefore, an important clinical consideration. It is also of considerable interest to public health.

Nucleic acid amplification tests (NAATs) are rapid, sensitive, and specific for the detection of $M$ tuberculosis complex DNA or RNA in respiratory specimens. ${ }^{7}$ NAATs have higher sensitivity than acid-fast or fluorochrome smears in detecting culture-positive samples because their detection threshold is as low as 1 organism per $100 \mathrm{~mL}$ of sample. They have a sensitivity and specificity greater than $95 \%$ in smear-positive specimens. ${ }^{8}$ However, in smear-negative samples the sensitivity of polymerase chain reaction (PCR) is between $40 \%$ and $77 \%$ but the specificity remains high. ${ }^{9}$ The wide range of sensitivity may be explained by the use of different samples such as bronchoalveolar lavage fluid (BALF) versus sputum. In fact, a recent study has disclosed that a combined use of BALF smears and $M$ tuberculosis NAATs has a good diagnostic yield in patients with sputum smear-negative TB or those without sputum production. ${ }^{10}$

Bronchoscopy with an acid-fast bacillus (AFB) smear and a culture for M tuberculosis of BALF have been introduced to 


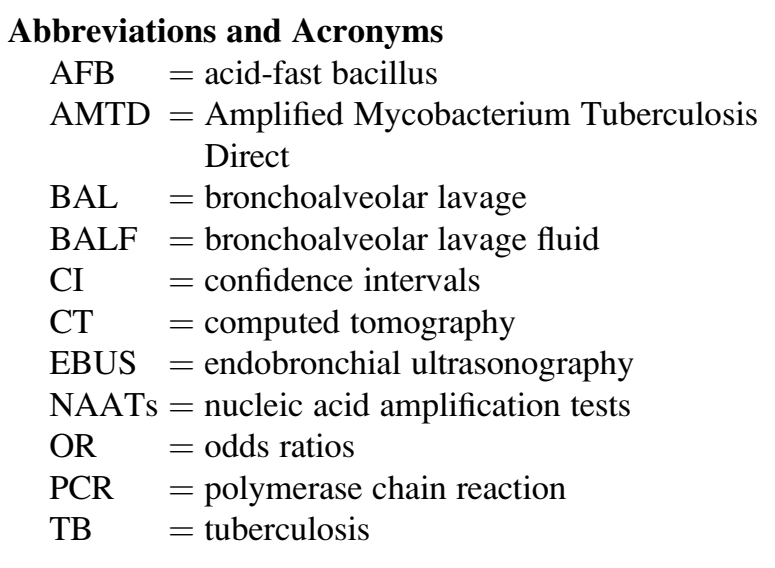

obtain a bacteriologic diagnosis of TB in smear-negative patients or patients unable to expectorate. ${ }^{11}$ Endobronchial ultrasonography (EBUS), a newly introduced technique, has also been applied to assess peripheral lung lesions. ${ }^{12}$ Compared with conventional bronchoscopy, EBUS offers the benefits of visualizing the parabronchial structure, confirming the precise location of peripheral lung lesions, and therefore improving the diagnostic yield of lung cancer. ${ }^{13,14}$ However, few studies have investigated the role of EBUS in the diagnosis of benign lung lesion. Our recent study has demonstrated that the addition of EBUS to a diagnostic bronchoscopy increases the incidence for proving the presence of TB in a population of patients with negative AFB smear or no sputum production. ${ }^{15}$ The study analyzed all subjects with pulmonary TB. Therefore, the sensitivity, specificity, and positive and negative predictive values could not be determined. In addition, the role of bronchoscopy with the aid of EBUS in the positive yield of NAATs for pulmonary TB in sputum-negative patients is still undetermined.

This study was designed to compare the diagnostic yields of AFB smear, NAATs, and $M$ tuberculosis culture in BALF for pulmonary TB by bronchoscopy, with or without EBUS, in patients with a negative sputum smear or without sputum production. The contributions of EBUS-guided procedures on the early diagnosis of pulmonary TB were also determined.

\section{PATIENTS AND METHODS}

\section{Study Subjects}

This study retrospectively analyzed patients who underwent bronchoscopy at Chang Gung Memorial Hospital, a university-affiliated, 3300-bed hospital in Taiwan. With the capability of performing Amplified Mycobacterium Tuberculosis Direct (AMTD) test since 2006, the institutional guideline recommended that patients with symptoms or chest radiographs suggestive of pulmonary TB may receive bronchoalveolar lavage (BAL) for AFB smear, $M$ tuberculosis culture, and AMTD test. Before introduction of the guideline, patients with suspected pulmonary TB received bronchoscopy for BAL and transbronchial lung biopsy. ${ }^{15}$ In contrast, no patients received biopsy in the present study. The inclusion criteria were symptoms or

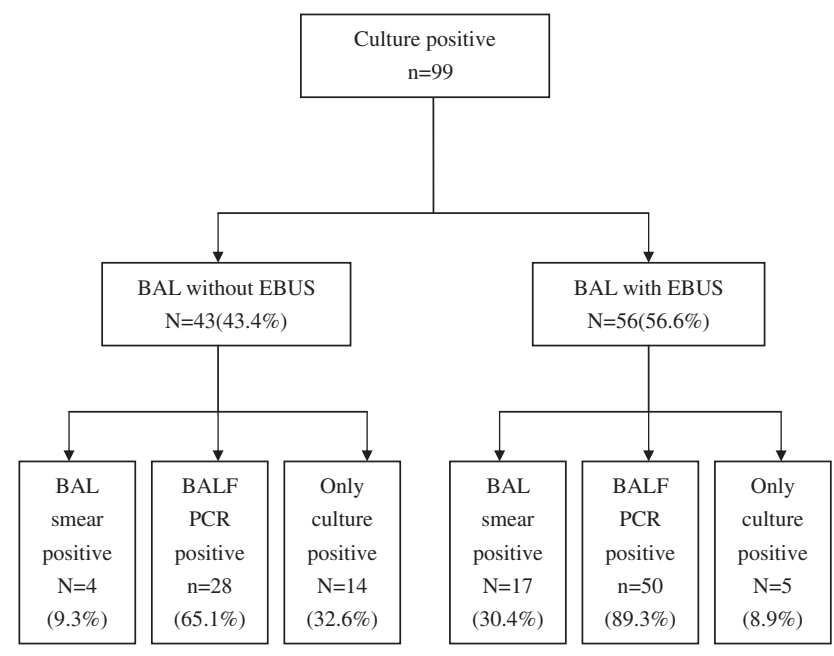

FIGURE 1. Analysis of patients with suspected pulmonary tuberculosis. $B A L$, Bronchoalveolar lavage; $B A L F$, bronchoalveolar lavage fluid; $B A L$ with EBUS, endobronchial ultrasound; $B A L$ without EBUS, conventional bronchoscopy; $P C R$, polymerase chain reaction.

chest radiographs suggestive of pulmonary TB in patients who received BAL because of their lack of spontaneous sputum or a negative AFB smear in 3 consecutive daily sputum specimens. The exclusion criteria were failure to receive a complete BAL procedure or sputum smears that were positive for AFB. From June 2006 to July 2007, a total of 347 patients with symptoms or chest radiographs suggestive of pulmonary TB were referred to our bronchoscopic center for BAL because of their lack of spontaneous sputum or a negative AFB smear in 3 consecutive daily sputum specimens (Figure 1). There are 2 bronchoscopy rooms in the bronchoscopic center but only 1 of them is equipped with the EBUS system. Patients referred to the bronchoscopic center received bronchoscopy with or without EBUS depending on the availability of the room equipped with the EBUS system. Among the 347 patients, 99 had a positive culture for $M$ tuberculosis in sputum or BALF, whereas the absence of $M$ tuberculosis in both BALF and sputum was found in the other 248 patients. Patients who received bronchoscopic examination with EBUS guidance were defined as the EBUS group. The other patients underwent bronchoscopic examinations during the time period when EBUS was not available. They received bronchoscopic examination without EBUS guidance and were designated as the non-EBUS group. The diagnostic yields of the BALF AFB smear, AMTD test, and culture for $M$ tuberculosis were compared between both groups. None of them was infected with human immunodeficiency virus. Informed consent to undergo bronchoscopy was obtained from all the subjects, and this study was approved by the hospital internal review board. Patients' baselines included age, gender, location and size of lesion, and radiologic appearance. Their underlying medical history was also collected, including diabetes mellitus, hypertension, history of malignancy, chronic airway obstructive disease, and previous TB infection.

\section{Procedure and Equipment of Bronchoscopy}

The bronchoscopic examinations were performed by experienced bronchoscopists who had performed more than 200 bronchoscopic procedures each year. To ensure the quality of EBUS in the study, we retrieved the EBUS group after each of the bronchoscopists had performed more than 50 EBUS procedures. The probable location of the lesion was determined initially by traditional posteroanterior chest radiography with and without chest computed tomography (CT). A flexible fiberoptic bronchoscope (BF-P240 or BF-40; Olympus, Tokyo, Japan) was then inserted via the nostril after anesthetizing with a local spray of $2 \%$ lidocaine (Xylocaine). In 
TABLE 1. Characteristics of 347 patients undergoing bronchoscopy

\begin{tabular}{lc}
\hline \multicolumn{1}{c}{ Variables } & No. $(\%)$ \\
\hline Age, y (mean \pm SD) & $54.9 \pm 12.8$ \\
Female gender (n, \%) & $166(47.8 \%)$ \\
Size of lesion, cm (mean \pm SD) & $3.7 \pm 1.9$ \\
Lobe distribution of lesions (n, \%) & \\
Right upper lobe & $145(41.8 \%)$ \\
Right middle lobe & $32(9.2 \%)$ \\
Right lower lobe & $28(8.1 \%)$ \\
Left upper lobe & $128(36.9 \%)$ \\
Left lower lobe & $14(4.0 \%)$ \\
Benign lesions (n, \%) & $213(61.4 \%)$ \\
Tuberculosis & $99(28.5 \%)$ \\
Pneumonia or other infection disease & $58(16.7 \%)$ \\
Fibrosis & $41(11.8 \%)$ \\
Organizing pneumonia & $15(4.3 \%)$ \\
Malignant lesions (n, \%) & $134(38.6 \%)$ \\
Non-small cell lung cancer & $121(34.9 \%)$ \\
Small cell lung cancer & $13(3.7 \%)$ \\
Metastatic cancer & $10(2.9 \%)$ \\
\hline
\end{tabular}

SD, Standard deviation.

patients who received bronchoscopy with EBUS, a 20-MHz radial mechanical type ultrasound probe (model UM-S20-S20R; Olympus) and an ultrasound unit (Endoscopic Ultrasound System; Olympus) were used in the procedure. To assess lesions in the periphery of the lung, we advanced the EBUS catheter into the fourth- to sixth-order bronchi. Once the lesion was located by EBUS, the neighboring bronchiolar orifices were also examined to ensure the precise location of lesions. Each EBUS image was evaluated for 3 features including the continuous margin of the lesion, the presence of an air bronchogram, and heterogeneous echogenicity of the internal structure. ${ }^{13}$ The EBUS procedure was performed without a guide sheath.

\section{Specimen Processing}

In the EBUS group, BAL was performed via the bronchiolar orifice determined by the EBUS image. In the conventional bronchoscopy group, the location of the lesion was determined by chest radiographs or CT and BAL was performed as far as the bronchoscope could advance. BAL was performed by infusion of 6 aliquots of $50 \mathrm{~mL}$ normal saline. ${ }^{16}$ The return was submitted for the AFB smear, AMTD tests, and culture. Lavaged specimens were processed by standard methods and stained with fluorochrome. ${ }^{11}$ The specimens were decontaminated with $N$-acetyl-L-cysteine$\mathrm{NaOH}$ (BBL MycoPrep; Becton Dickinson, Cockeysville, Md). Positive smears were confirmed by Ziehl-Neelsen staining. Specimens were cultured with BACTEC 12B vials (radiometric BACTEC 460TB system; Becton Dickinson, Franklin Lakes, NJ) and Lowenstein-Jensen media (Bio-Rad, Marnesla-Coquette, France). PCR testing for $M$ tuberculosis complex was performed with the AMTD test kit (Gen-Probe, San Diego, Calif) according to the instructions of the manufacturer. ${ }^{17}$ The specimen results were read in a Leader 450 luminometer (Gen-Probe); a cutoff value of 30,000 relative light units or more was used for diagnosing positive specimens. Positive and negative controls were included in every run. The primary diagnosis of TB was made by a positive AFB smear or NAATs in BALF; the definitive diagnosis was confirmed by the presence of $M$ tuberculosis cultured in sputum or BALF. The treatment of TB was started if a positive AFB smear or NAATs in BALF were obtained. For those with a negative AFB smear and NAATs in BALF, anti-TB treatment was started until the results of positive $M$ tuberculosis cultured in sputum or BALF were obtained. The time to diagnosis and treatment of TB was measured from the patient's first visit to the medical center to the diagnosis and treatment of TB.

\section{Statistical Analysis}

Data are expressed as either group percentages (categorical variables) or mean \pm standard deviation (continuous variables). Data were compared between patients who received bronchoscopy with versus without EBUS. Categorical variables were compared by $\chi^{2}$ or Fisher's exact test. Odds ratios (OR) and their $95 \%$ confidence intervals (CI) were used to assess the difference of diagnostic yields between the 2 groups. We applied the $t$ test to compare continuous variables with normal distribution and the Mann-Whitney test for continuous variables not distributed normally $(P<.05$ for all). All analyses were performed with SPSS software version 10.0 (SPSS, Inc, Chicago, Ill)

\section{RESULTS \\ Patient Characteristics}

A total of 347 patients were analyzed in the study (Table 1). In 99 patients TB was diagnosed owing to the presence of $M$ tuberculosis cultured in sputum or BALF. The group comprised 166 women and 181 men with a mean age of $54.9 \pm 12.8$ years. The mean lesion size was $3.7 \pm 1.9 \mathrm{~cm}$. Two hundred thirteen patients had benign lesions and 134 patients had malignant lesions. The baseline characteristics of patients with culture-confirmed TB are summarized in Table 2. There were no significant differences between groups regarding demographic variables, size of lesion, percentage of retrieved BALF, chest CT scan, location of lesion, radiologic appearance, and chronic coexisting conditions. For the EBUS groups, 51 (91.1\%) lesions could be localized by EBUS. The presence of air bronchograms was observed in $44(78.6 \%)$ patients; lesions with a complete margin and homogeneous echogenicity were detected in $22(39.3 \%)$ and $40(71.4 \%)$ patients, respectively. Diabetes mellitus was the most frequent chronic comorbidity in the 2 groups. The overall mean time for procedure was $11.1 \pm 2.3$ minutes for the EBUS group and $9.7 \pm$ 2.3 minutes for the non-EBUS group.

\section{Sensitivity, Specificity, and Predictive Values of BAL for the Diagnosis of Pulmonary TB}

Sensitivities, specificities, and predictive values of AFB smear and NAATs for the diagnosis of culture confirmed pulmonary TB in BALF are shown in Table 3 . The sensitivity of AFB smear in BALF was $30.4 \%$ in the EBUS group and $9.3 \%$ in the non-EBUS group. The positive predictive value was $85 \%$ in the EBUS group and $66.7 \%$ in the non-EBUS group. The specificities and negative predictive values were similar in both groups. The sensitivity of NAATs in BALF was $89.3 \%$ in the EBUS group and $65.1 \%$ in the non-EBUS group. The specificities and positive and negative predictive values were similar in the 2 groups.

\section{The Yield of Sputum and Lavage Specimen for Culture-Confirmed TB Diagnosis}

The diagnostic yields of the NAATs in BALF $(89.3 \%, 50$ / 56; OR, 4.46; 95\% CI, 1.56-12.81; $P=.006$ ), AFB smear in BALF $(30.4 \%, 17 / 56$; OR, 4.25; 95\% CI, 1.31-13.78; 
TABLE 2. The characteristics of patients with culture-confirmed pulmonary tuberculosis

\begin{tabular}{|c|c|c|c|}
\hline Baseline characteristics & $\begin{array}{c}\text { Without } \\
\text { EBUS }(n=43)\end{array}$ & $\begin{array}{l}\text { With EBUS } \\
(\mathbf{n}=\mathbf{5 6})\end{array}$ & $\begin{array}{c}P \\
\text { value }\end{array}$ \\
\hline Age, y (mean \pm SD) & $48.4 \pm 21.9$ & $53.6 \pm 19$ & .210 \\
\hline Female sex, no. $(\%)$ & $12(27.9 \%)$ & $20(35.7 \%)$ & .517 \\
\hline Size of lesion, $\mathrm{cm}($ mean $\pm \mathrm{SD})$ & $3.8 \pm 1.9$ & $4.3 \pm 1.7$ & .174 \\
\hline Percentage of retrieved BALF & $39.2 \pm 22$ & $39.4 \pm 19.8$ & .535 \\
\hline $\begin{array}{c}\text { Lesion localized by } \\
\text { EBUS, no. }(\%)\end{array}$ & NA & $51(91.1 \%)$ & NA \\
\hline With computed tomography & $14(32.6 \%)$ & $26(42.4 \%)$ & .216 \\
\hline \multicolumn{4}{|l|}{ Location of lesion } \\
\hline Right upper lobe & $21(48.8 \%)$ & $22(39.3 \%)$ & .415 \\
\hline Right middle lobe & $4(9.3 \%)$ & $6(10.7 \%)$ & 1.00 \\
\hline Right lower lobe & $1(2.3 \%)$ & $6(10.7 \%)$ & .135 \\
\hline Left upper lobe & $17(39.5 \%)$ & $20(35.7 \%)$ & .834 \\
\hline Left lower lobe & $0(0 \%)$ & $2(4.7 \%)$ & .504 \\
\hline \multicolumn{4}{|l|}{ Radiologic appearance } \\
\hline Alveolar consolidation & $18(41.9 \%)$ & $22(39.3 \%)$ & .838 \\
\hline Fibronodular infiltration & $16(37.2 \%)$ & $20(35.7 \%)$ & 1.00 \\
\hline Cavity & $6(14 \%)$ & $8(14.4 \%)$ & 1.00 \\
\hline Multiple nodules or mass & $4(9.3 \%)$ & $7(12.5 \%)$ & .752 \\
\hline With lymphadenopathy & $4(9.3 \%)$ & $3(5.4 \%)$ & .464 \\
\hline Miliary shadowing & $2(4.7 \%)$ & $1(1.8 \%)$ & .578 \\
\hline Lower lung field TB & $1(2.3 \%)$ & $3(5.4 \%)$ & .631 \\
\hline \multicolumn{4}{|l|}{ Echoic features by EBUS } \\
\hline Presence of air bronchogram & NA & $44(78.6 \%)$ & NA \\
\hline Complete margin & NA & $22(39.3 \%)$ & NA \\
\hline Homogenous echogenicity & NA & $40(71.4 \%)$ & NA \\
\hline \multicolumn{4}{|l|}{$\begin{array}{l}\text { Chronic coexisting } \\
\quad \text { condition, no. }(\%)\end{array}$} \\
\hline Diabetes mellitus & $6(14 \%)$ & $8(14.3 \%)$ & 1.00 \\
\hline Hypertension & $6(14 \%)$ & $5(8.9 \%)$ & .525 \\
\hline Chronic renal insufficiency & $5(11.6 \%)$ & $2(4.7 \%)$ & .234 \\
\hline Cancer & $0(0 \%)$ & $2(4.7 \%)$ & .504 \\
\hline COPD & $5(11.6 \%)$ & $4(7.1 \%)$ & 496 \\
\hline Previous TB infection & $1(2.3 \%)$ & $2(4.7 \%)$ & 1.00 \\
\hline
\end{tabular}

EBUS, Endobronchial ultrasound; $S D$, standard deviation; $T B$, tuberculosis; $C O P D$, chronic obstructive pulmonary disease; $N A$, not available.

$P=.013)$, and $M$ tuberculosis in BALF culture $(67.9 \%, 38 /$ 56; OR, 2.43 ; 95\% CI, $1.07-5.53 ; P=.041)$ were significantly higher in EBUS patients. Diagnostic yields in those who underwent conventional bronchoscopy were $65.1 \%$ (28/43), 9.3\% (4/43), and 46.5\% (20/43), respectively (Table 4). In contrast, similar incidences of positive sputum M tuberculosis culture were found in both groups. There was a higher proportion of patients in the conventional group whose TB diagnosis was documented only by positive culture $(32.6 \%$, either from BALF or sputum) than among EBUS patients $(8.9 \% ; P=.004)$ (Figure 1). Combining BALF smear and NAATs allowed a rapid diagnosis of pulmonary TB to be made in $51(91.1 \%)$ of the 56 EBUS patients and $29(67.4 \%)$ of the 43 remaining patients who received conventional bronchoscopy. With the aid of EBUS, the overall diagnostic yield for TB by bronchoscopic procedures (smear, NAATs, and culture of BALF) was higher $(92.9 \%)$ than that for those who did not undergo EBUS (74.4\%; OR, 4.47; 95\% CI, 1.31-15.23; $P=.021)$. In contrast, pulmonary TB was diagnosed only by sputum culture of $M$ tuberculosis in $4(7.1 \%)$ of the 56 EBUS patients and $11(25.6 \%)$ of the 43 non-EBUS patients.

\section{The Effects of CT, Location, and Size of Lesion on NAATs Yields}

The use of EBUS in bronchoscopy had higher diagnostic yields of NAATs than conventional bronchoscopy in patients with $(22 / 26,84.6 \%$ vs $7 / 14,50 \%$; OR, $5.5 ; 95 \%$ CI, $1.23-24.52 ; P=.029)$ or without $(28 / 30,93.3 \%$ vs 21/29, 72.4\%; OR, 5.33; 95\% CI, 1.02-27.77; $P=.042$ ) chest $\mathrm{CT}$ scan before the bronchoscopic procedures. In patients in whom the lesion was smaller than $3 \mathrm{~cm}$ in size, bronchoscopy with EBUS $(\mathrm{n}=40)$ had a higher diagnostic yield of NAATs $(36 / 40,90 \%$ vs $15 / 23,65.2 \%$; OR, 4.8 ; $95 \%$ CI $1.25-18.39 ; P=.022)$ in BALF than conventional bronchoscopy $(n=23)$. Despite the lack of statistical significance, bronchoscopy with EBUS $(n=16)$ seemed to have a higher yield of NAATs $(14 / 16,87.5 \%$ vs $15 / 23,65 \%$; OR, $3.8 ; 95 \% \mathrm{CI}, 0.66-21.56 ; P=.245)$ in BALF compared with conventional bronchoscopy $(\mathrm{n}=20)$ in those in whom the lesion was larger than $3 \mathrm{~cm}$ in size.

\section{The Treatment of Pulmonary TB}

The time to diagnosis of TB in EBUS patients (18.6 \pm 18.4 days) was shorter than that in non-EBUS patients (28.7 \pm 24.7 days; $P=.022$ ). Similarly, the time to initiate treatment in patients receiving bronchoscopy with EBUS (26.0 \pm 17.7 days; $P=.013$ ) was shorter than that in patients receiving conventional bronchoscopy ( $36.8 \pm 24.6$ days). In our study, most of the patients received bronchoscopy in the outpatient clinic rather than during hospitalization. Therefore, the time lag between diagnosis of TB and initiation of treatment may be due to the schedule of the next clinic visit.

\section{Complications}

Pneumothorax occurred in $2(1.2 \%)$ patients of the conventional bronchoscopy group and resolved spontaneously, but no pneumothorax developed after the EBUS procedure. No major bleeding with hemodynamic instability developed during the procedure in either group.

\section{DISCUSSION}

This study has demonstrated that the introduction of EBUS increases the diagnostic yield of the NAATs, AFB smear, and $M$ tuberculosis culture from BALF in patients with pulmonary TB who have a negative sputum smear or no spontaneous sputum production. Combining the BALF smear and NAATs, bronchoscopy with EBUS allows for 
TABLE 3. Sensitivity, specificity, and predictive values of BALF smear and AMTD test for the diagnosis of culture confirmed pulmonary TB

\begin{tabular}{|c|c|c|c|c|c|c|}
\hline \multirow[b]{2}{*}{ Procedure } & \multicolumn{2}{|c|}{ Final diagnosis (no. positive/tested) } & \multirow[b]{2}{*}{ Sensitivity $(\%)$} & \multirow[b]{2}{*}{ Specificity $(\%)$} & \multirow{2}{*}{$\begin{array}{c}\text { Positive predictive } \\
\text { value }(\%) \\
\end{array}$} & \multirow{2}{*}{$\begin{array}{c}\text { Negative predictive } \\
\text { value }(\%)\end{array}$} \\
\hline & TB & Non-TB & & & & \\
\hline \multicolumn{7}{|l|}{ BALF smear } \\
\hline With EBUS & $17 / 56$ & $3 / 130$ & 30.4 & 97.7 & 85 & 76.5 \\
\hline Without EBUS & $4 / 43$ & $2 / 118$ & 9.3 & 98.3 & 66.7 & 74.8 \\
\hline \multicolumn{7}{|l|}{ BALF AMTD } \\
\hline With EBUS & $50 / 56$ & $5 / 130$ & 89.3 & 96.2 & 90.9 & 95.4 \\
\hline Without EBUS & $28 / 43$ & $3 / 118$ & 65.1 & 97.5 & 90.3 & 95.4 \\
\hline
\end{tabular}

$B A L F$, Bronchoalveolar lavage fluid; $A M T D$, Amplified Mycobacterium Tuberculosis Direct; $T B$, tuberculosis; EBUS, endobronchial ultrasound.

a higher rate of rapid diagnosis of TB than conventional bronchoscopy in those patients. In addition, the time to diagnosis and time to treatment of TB in EBUS patients was shorter than that in non-EBUS patients.

The clinical application of EBUS began in the early 1990s. ${ }^{12}$ EBUS was documented in improving the diagnostic yield for lung cancer; the EBUS image pattern might offer clues for differentiating benign lesions from malignant peripheral lung lesions. ${ }^{13}$ Apart from its role in the diagnosis of lung cancer, our previous study had shown that EBUS increased the diagnostic yield of BAL and transbronchial lung biopsy in TB patients with negative sputum smear. ${ }^{15}$ However, that study analyzed all subjects with pulmonary TB; therefore, the sensitivity, specificity, and positive and negative predictive values could not be determined. The present study recruited patients with symptoms or chest radiographs suggestive of pulmonary TB. In 99 of the 347 patients, TB was diagnosed owing to the presence of positive $M$ tuberculosis culture. The present study provided the sensitivity, specificity, and positive and negative predictive value of BAL smear and NAATs in the diagnosis of pulmonary TB. A recent study has shown the good diagnostic yield for pulmonary TB by NAATs in BALF obtained by conventional bronchoscopy. ${ }^{10}$ Our study further disclosed that EBUS increased the sensitivity of NAATs in BALF for the diagnosis of pulmonary $\mathrm{TB}$ in patients with sputum smear-negative condition or those without sputum production.

The sputum-negative conditions in patients with pulmonary TB have been reported to be the major causes of treatment delay for TB. ${ }^{5}$ To avoid delay in treatment, physicians have been using fiberoptic bronchoscopy for obtaining lower respiratory tract sections in those patients. ${ }^{18}$ Once the lesion is not visible by conventional bronchoscopy, BAL is performed in a best-guess fashion or with the aid of a fluoroscope.${ }^{19}$ In the lesions that are not visualized by conventional bronchoscopy, EBUS images allow confirmation of the precise location of lesions by direct visualization. Therefore, with the aid of EBUS to accurately localize the lesion, bronchoscopy-guided BAL for AFB smear and NAATs increased the overall diagnostic yield for pulmonary TB to more than $90 \%$. Pneumothorax is an unusual complication of BAL. The development of pneumothorax after BAL had been reported in patients with obstructive airway disease. ${ }^{20}$ Compatible with a previous report, our study showed that pneumothorax developed after BAL in 2 patients with chronic obstructive pulmonary disease.

When conventional bronchoscopy is performed, knowledge of the $\mathrm{CT}$ appearance may improve the diagnostic yield of lung cancer by better targeting suspicious lesions. This applies to both endobronchial diseases visible with the bronchoscope with transbronchial biopsy specimens and washings taken from the periphery of the lung. ${ }^{21}$ However, our study demonstrated that the higher diagnostic yield of NAATs in EBUS groups than the non-EBUS group was unaffected by having a CT scan or not. This discrepancy could be caused by the difference of disease (TB in our study versus lung cancer in previous studies) and the different procedures used in these studies (NAATs in BALF in our study versus transbronchial lung biopsy and washing in previous studies). In addition, bronchoscopy with EBUS had a higher

TABLE 4. Comparison of results of AMTD, AFB smears, and M tuberculosis cultures in BALF and sputum from patients with culture confirmed pulmonary TB

\begin{tabular}{|c|c|c|c|c|c|}
\hline & With EBUS $(n=56)$ & Without EBUS $(n=43)$ & OR & $95 \% \mathbf{C I}$ & $P$ value \\
\hline Positive BALF AMTD & $50(89.3 \%)$ & $28(65.1 \%)$ & 4.46 & $1.56-12.81$ & .006 \\
\hline Positive AFB smear of BALF & $17(30.4 \%)$ & $4(9.3 \%)$ & 4.25 & $1.31-13.78$ & .013 \\
\hline Positive BALF M tuberculosis culture & $38(67.9 \%)$ & $20(46.5 \%)$ & 2.43 & $1.07-5.53$ & .041 \\
\hline Positive sputum $M$ tuberculosis culture & $24(42.9 \%)$ & $24(55.8 \%)$ & 0.59 & $0.27-1.32$ & .228 \\
\hline Positive BALF AMTD and/or AFB smear & $51(91.1 \%)$ & $29(67.4 \%)$ & 4.92 & $1.61-15.07$ & .004 \\
\hline Positive BALF smear, AMTD, or culture & $52(92.9 \%)$ & $32(74.4 \%)$ & 4.47 & $1.31-15.23$ & .021 \\
\hline
\end{tabular}

$A M T D$, Amplified Mycobacterium Tuberculosis Direct; $A F B$, acid-fast bacillus; $B A L F$, bronchoalveolar lavage fluid; $T B$, tuberculosis; $E B U S$, endobronchial ultrasound; $O R$, odds ratio; $C I$, confidence interval. 
diagnostic yield of NAATs in BALF than conventional bronchoscopy, especially in lesions less than $3 \mathrm{~cm}$ in diameter. In those patients with small-sized lesions, it was difficult to obtain the diagnosis while pulmonary TB was suspected. Our results provide the evidence for the usefulness of bronchoscopy with EBUS in the diagnosis for TB among patients with small-sized lesions. Owing to the small sample size in the subgroup with a lesion size of less than $3 \mathrm{~cm}$, the role of EBUS in the diagnostic yield of NAATs deserves further investigation.

The value of NAATs in respiratory samples to obtain a rapid diagnosis of pulmonary $\mathrm{TB}$ has been described in many studies. ${ }^{10,22}$ A positive NAAT result may be valuable in the early detection of active TB cases that are smear-negative. ${ }^{23}$ One previous study reported a sensitivity of $53 \%$ and a specificity of $93 \%$ in comparison with the culture when NAAT was applied to smear-negative specimens ${ }^{24}$; this suggests that a potentially significant number of cases of smear-negative TB could be quickly detected with the expanded use of NAATs. In a patient with clinically suspected TB who has a negative AFB smear and a positive NAAT, therapy can be started sooner and contact investigation can be initiated. ${ }^{25}$ By combined use of bronchoscopy and NAATs, Tueller and associates ${ }^{10}$ have reported that NAATs in BALF had a sensitivity of $78 \%$ for detection of pulmonary TB in sputum smear-negative patients or patients without sputum production. Our results have shown that the NAATs of BALF offered rapid evidence for TB infection in $89.3 \%$ of the patients in the EBUS group, but only in $65.1 \%$ of the patients who received conventional bronchoscopy. Combining AFB smear and NAATs in BALF as the rapid evidence for diagnosis, only $8.9 \%$ of the patients in the EBUS group needed to wait until a positive culture as the only evidence for TB diagnosis. In contrast, $32.6 \%$ of the patients in the conventional group had culture as the only clue for TB diagnosis. The high diagnostic yield of EBUS-guided bronchoscopy for early diagnosis of TB infection may therefore avoid a delay in administration of anti-TB treatment or the need for further invasive procedures such as CT-guided or surgical biopsy to reach a definite diagnosis.

Some limitations of this study need to be mentioned. First, results of BALF smear and NAATs may be false positive; therefore, $M$ tuberculosis cultures in BALF or sputum are still required for a definite diagnosis. In addition, the study only recruited patients with negative AFB smear or no sputum production. Therefore, the results of our study could not be extended to all the patients with suggestive TB lesions.

In conclusion, sputum AFB smears and $M$ tuberculosis cultures should be performed first in patients in whom pulmonary TB is suspected. Once a negative sputum AFB smear or no spontaneous sputum production occurs, the introduction of EBUS increases the diagnostic yield of the NAATs, AFB smear, and $M$ tuberculosis culture from
BALF. By means of a BALF smear and NAATs, bronchoscopy with EBUS allows for a higher rate of rapid diagnosis of TB than conventional bronchoscopy in patients with negative sputum smear or no spontaneous sputum production.

\section{References}

1. Sputum-smear-negative pulmonary tuberculosis: controlled trial of 3-month and 2-month regimens of chemotherapy. Lancet. 1979;1(8131):1361-3.

2. Watterson SA, Drobniewski FA. Modern laboratory diagnosis of mycobacterial infections. J Clin Pathol. 2000;53:727-32.

3. Rohner P, Ninet B, Metral C, Emler S, Auckenthaler R. Evaluation of the MB/ BacT system and comparison to the BACTEC 460 system and solid media for isolation of mycobacteria from clinical specimens. J Clin Microbiol. 1997;35: 3127-31.

4. Diagnostic Standards and Classification of Tuberculosis in Adults and Children. This official statement of the American Thoracic Society and the Centers for Disease Control and Prevention was adopted by the ATS Board of Directors, July 1999. This statement was endorsed by the Council of the Infectious Disease Society of America, September 1999. Am J Respir Crit Care Med. 2000;161(4 Pt 1): 1376-95.

5. Mathur P, Sacks L, Auten G, Sall R, Levy C, Gordin F. Delayed diagnosis of pulmonary tuberculosis in city hospitals. Arch Intern Med. 1994;154:306-10.

6. Pablos-Mendez A, Sterling TR, Frieden TR. The relationship between delayed or incomplete treatment and all-cause mortality in patients with tuberculosis. JAMA. 1996;276:1223-8.

7. Bradley SP, Reed SL, Catanzaro A. Clinical efficacy of the amplified Mycobacterium tuberculosis direct test for the diagnosis of pulmonary tuberculosis. Am J Respir Crit Care Med. 1996;153:1606-10.

8. Abe C, Hirano K, Wada M, Kazumi Y, Takahashi M, Fukasawa Y, et al. Detection of Mycobacterium tuberculosis in clinical specimens by polymerase chain reaction and Gen-Probe Amplified Mycobacterium Tuberculosis Direct Test. $J$ Clin Microbiol. 1993;31:3270-4.

9. Bogard M, Vincelette J, Antinozzi R, Alonso R, Fenner T, Schirm J, et al. Multicenter study of a commercial, automated polymerase chain reaction system for the rapid detection of Mycobacterium tuberculosis in respiratory specimens in routine clinical practice. Eur J Clin Microbiol Infect Dis. 2001;20: 724-31.

10. Tueller C, Chhajed PN, Buitrago-Tellez C, Frei R, Frey M, Tamm M. Value of smear and PCR in bronchoalveolar lavage fluid in culture positive pulmonary tuberculosis. Eur Respir J. 2005;26:767-72.

11. Willcox PA, Benatar SR, Potgieter PD. Use of the flexible fibreoptic bronchoscope in diagnosis of sputum-negative pulmonary tuberculosis. Thorax. 1982; 37:598-601.

12. Hurter T, Hanrath P. Endobronchial sonography: feasibility and preliminary results. Thorax. 1992;47:565-7.

13. Kuo CH, Lin SM, Chen HC, Chou CL, Yu CT, Kuo HP. Diagnosis of peripheral lung cancer with three echoic features via endobronchial ultrasound. Chest. 2007; 132:922-9.

14. Yang MC, Liu WT, Wang CH, Lin HC, Chen HC, Chou CL, et al. Diagnostic value of endobronchial ultrasound-guided transbronchial lung biopsy in peripheral lung cancers. J Formos Med Assoc. 2004;103:124-9.

15. Lin SM, Chung FT, Huang CD, Liu WT, Kuo CH, Wang CH, et al. Diagnostic value of endobronchial ultrasonography for pulmonary tuberculosis. $J$ Thorac Cardiovasc Surg. 2009;138:179-84.

16. Wang CH, Liu CY, Wan YL, Chou CL, Huang KH, Lin HC, et al. Persistence of lung inflammation and lung cytokines with high-resolution CT abnormalities during recovery from SARS. Respir Res. 2005;6:42.

17. Miller N, Hernandez SG, Cleary TJ. Evaluation of Gen-Probe Amplified Mycobacterium Tuberculosis Direct Test and PCR for direct detection of Mycobacterium tuberculosis in clinical specimens. J Clin Microbiol. 1994;32:393-7.

18. Chawla R, Pant K, Jaggi OP, Chandrashekhar S, Thukral SS. Fibreoptic bronchoscopy in smear-negative pulmonary tuberculosis. Eur Respir J. 1988;1: 804-6.

19. Yoshikawa M, Sukoh N, Yamazaki K, Kanazawa K, Fukumoto S, Harada M, et al. Diagnostic value of endobronchial ultrasonography with a guide sheath for peripheral pulmonary lesions without X-ray fluoroscopy. Chest. 2007;131: 1788-93.

20. Ruiz F, Casado T, Monso E. Pneumothorax during bronchoalveolar lavage. Chest. 1989;96:1441-2. 
21. Laroche C, Fairbairn I, Moss H, Pepke-Zaba J, Sharples L, Flower C, et al. Role of computed tomographic scanning of the thorax prior to bronchoscopy in the investigation of suspected lung cancer. Thorax. 2000;55:359-63.

22. Querol JM, Farga MA, Granda D, Gimeno C, Garcia-de-Lomas J. The utility of polymerase chain reaction $(\mathrm{PCR})$ in the diagnosis of pulmonary tuberculosis. Chest. 1995;107:1631-5.

23. Rapid diagnostic tests for tuberculosis: what is the appropriate use? American Thoracic Society Workshop. Am J Respir Crit Care Med. 1997;155:1804-14.
24. Cohen RA, Muzaffar S, Schwartz D, Bashir S, Luke S, McGartland LP, et al. Diagnosis of pulmonary tuberculosis using PCR assays on sputum collected within 24 hours of hospital admission. Am J Respir Crit Care Med. 1998;157: 156-61.

25. Lim TK, Mukhopadhyay A, Gough A, Khoo KL, Khoo SM, Lee KH, et al. Role of clinical judgment in the application of a nucleic acid amplification test for the rapid diagnosis of pulmonary tuberculosis. Chest. 2003;124: 902-8. 